\title{
La prescripción inmediata de antibióticos para las infecciones no complicadas del tracto respiratorio inferior no reducirían las hospitalizaciones ni la mortalidad
}

Prescribing antibiotics immediately for uncomplicated infections of the lower respiratory tract does not reduce hospital admission or mortality.

Little P y col. BMJ 2017;357: j2148. PMID: 28533265

\section{Comentado de:}

Little P y col. BMJ 2017;357: j2148. PMID: $28533265^{1}$

\section{Objetivos}

Evaluar el impacto en los resultados adversos de diferentes estrategias de prescripción de antibióticos para tratar infecciones del tracto respiratorio inferior (ITRI) no complicadas en una población de 16 años o más de edad.

\section{Diseño, población y lugar}

Estudio de tipo cohorte prospectiva realizado en el primer nivel de atención del Reino Unido. Se incluyeron pacientes de 16 años o más que presenten una ITRI, definida como tos aguda (de tres semanas o menos de evolución) como síntoma principal y de origen infeccioso, determinada por el médico. Se excluyeron los pacientes que tenían otras causas de tos (por ejemplo, insuficiencia cardíaca, reflujo gastroesofágico, alveolitis fibrosante), quienes no fueron capaces de dar su consentimiento (por ejemplo, enfermedad mental grave), inmunodeprimidos, quienes presentaron previamente el mismo episodio de enfermedad, o aquellos que hubieran sido incluidos anteriormente.

\section{Medición de resultados principales}

Los médicos completaron un formulario de registro clínico en la consulta que incluyó datos como edad, historia de tabaquismo, duración y naturaleza de los síntomas, resultados del examen físico, una puntuación de severidad global, si se prescribieron antibióticos o no, y si fue de forma inmediata o diferida.

Los resultados principales fueron: nueva consulta con síntomas de ITRI en los 30 días posteriores a la consulta, hospitalización o muerte; y fueron evaluados a través de la revisión de historias clínicas a los 30 días. Se realizó un análisis utilizando un método de puntaje de propensión estratificado comparando cada estrategia de prescripción contra un abordaje inicial sin uso de antibióticos (es decir, antibióticos inmediatos vs. no uso de antibióticos, y antibióticos diferidos vs no uso de antibióticos).

\section{Resultados}

Se resumen en la Tabla 1 . Se incluyeron 2.886 participantes. Se excluyeron los pacientes con diagnóstico de neumonía o cáncer de plumón al inicio del estudio.

Tabla 1. Riesgo de hospitalización o muerte y reconsulta en pacientes con infección del tracto respiratorio inferior seguún la estrategia de prescripción de antibióticos. Notas: RR: riesgo relativo multivariado ajustado utilizando puntaje de propensión estratificado; IC $95 \%$ : intervalo de confianza del $95 \%$.

\begin{tabular}{|c|c|c|c|c|c|c|}
\hline \multirow{2}{*}{$\begin{array}{l}\text { Prescripción de } \\
\text { antibióticos }\end{array}$} & \multicolumn{2}{|c|}{ Hospitalización o muerte } & \multirow{2}{*}{$\begin{array}{l}\text { RR } \\
\text { (IC 95\%) } \\
\end{array}$} & \multicolumn{2}{|c|}{ Reconsulta } & \multirow{2}{*}{$\begin{array}{l}\text { RR } \\
\text { (IC 95\%) }\end{array}$} \\
\hline & $\begin{array}{c}\text { No }(n=28.583) \\
n(\%)\end{array}$ & $\begin{array}{c}\text { Si }(n=196) \\
n(\%)\end{array}$ & & $\begin{array}{c}\text { No }(n=22.343) \\
n(\%)\end{array}$ & $\begin{array}{c}\mathrm{Si}(\mathrm{n}=6.436) \\
\mathrm{n}(\%)\end{array}$ & \\
\hline Ninguna & $7.306(25,6)$ & $26(13,3)$ & 1,00 & $5.889(26,4)$ & $1.443(22,4)$ & 1,00 \\
\hline Inmediata & $17.472(61,1)$ & $156(79,6)$ & $1,06(0,63$ a 1,81$)$ & $13.173(59)$ & $4.455(69,2)$ & $0,98(0,90$ a 1,07$)$ \\
\hline Diferida & $3.805(13,3)$ & $14(7,1)$ & $0,81(0,41$ a 1,64$)$ & $3.281(14,7)$ & $538(8,4)$ & $0,64(0,57$ a 0,72$)$ \\
\hline
\end{tabular}

\section{Conclusiones}

En este estudio no fue posible demostrar que los antibióticos, prescriptos de forma inmediato o diferida, se asociaran con una reducción significativa en la hospitalización o muerte. La falta de resolución de los síntomas o la progresión de la enfermedad con síntomas nuevos fue frecuente, y la prescripción diferida de anti- bióticos, no así la inmediata, se asoció con una reducción significativa de las reconsultas.

Fuentes de Financiamiento : Beca del Instituto Nacional de Investigación en Salud (NIHR, por sus siglas en inglés)

Conflicto de interés de los autores: : No presenta.

\section{Comentario}

La Genómica para Combatir la Resistencia contra los Antibióticos para la infección adquirida en las vías respiratorias bajas de Europa (GRACE, por sus siglas en inglés) realizó un estudio multicéntrico donde no demostró ninguna ventaja clara la admi- 
nistración de antibióticos para el tratamiento de las $|T R|^{2}$. Conclusiones similares se desprenden de una revisión Cochrane actualizada sobre este mismo tema ${ }^{3}$. Sin embargo en estos trabajos no se mostraron los efectos como hospitalizaciones ni muertes, ya que al ser un evento infrecuente, no contaban con poder suficiente para hacerlo. Este último punto resulta quizá de particular importancia para reasegurar al médico a la hora de prescribir o no antibióticos cuando se temen consecuencias médico legales, sabiendo que la mayoría de las infecciones son realmente autolimitadas y que al indicar antibióticos incurrimos además en costos adicionales, efectos adversos potenciales, aumento de la resistencia antibiótica y aumento de las reconsultas.

El estudio aquí resumido presenta como fortaleza un gran número de pacientes y un poder estadístico suficiente para evaluar los eventos infrecuentes como muertes y hospitalizaciones. A su vez presenta un bajo riesgo de sesgo por los ajustes utilizados.

Entre las limitaciones se destacan que la prescripción no era ciega, lo que podría haber alterado el patrón de reconsulta; la existencia de un menor número de pacientes en la rama antibióticos respecto a la rama no antibióticos, que podría afectar los resultados; que la intervención podría no resultar extrapolable a nuestra realidad, ya que habitualmente no indicamos recetas de forma retrasada en nuestra práctica clínica; y que no se detalla un consenso sobre la prescripción de antibióticos sin embargo esto suele ocurrir habitualmente en nuestra práctica clínica.

\section{Conclusiones del comentador}

Según el estudio evaluado, los antecedentes y la baja incidencia del evento estudiado (muerte u hospitalización) no sería recomendable indicar antibióticos en las infecciones respiratorias bajas no complicadas.

María Florencia Herrera Bornes [ Hospital Italiano de Buenos Aires, Servicio de Medicina Familiar y Comunitaria florencia.herrera@hospitalitaliano.org. ar ]

Herrera Bornes MF La prescripción inmediata de antibióticos para las infecciones no complicadas del tracto respiratorio inferior no reducirían las hospitalizaciones ni la mortalidad. Evid Actual Pract Ambul. 2019;22(4):e001094. Comentado de: Little P, Stuart B, Smith S, Thompson MJ, Knox K, van den Bruel A. y col. Antibiotic prescription strategies and adverse outcome for uncomplicated lower respiratory tract infections: prospective cough complication cohort (3C) study BMJ 2017; 357 :j2148. PMID: 28533265

\section{Referencias}

1. Little P, Stuart B, Smith S, Thompson MJ, Knox K, VanDenBruel A, et al. Antibiotic prescription strategies and adverse outcome for uncomplicated lower respiratory tract infections: prospective cough complication cohort (3C) study. BMJ. 2017;357:2148-2148.

2. Little P, Stuart B, Moore M, Coenen S, Butler CC, Godycki-Cwirko M, et al. Amoxicillin for acute lower-respiratory-tract infection in primary care when pneumonia is not suspected: A 12-country, randomised, placebo-controlled trial. The Lancet Infectious Diseases. 2013;Available from: 10.1016/ S1473-3099(12)70300-6.

3. Smith SM, Fahey T, Smucny J, Becker LA. Antibiotics for acute bronchitis. Cochrane Database of Systematic Reviews. 2017;CD000245. Available from: 10.1002/14651858.CD000245.pub4. 